2nd International Conference on Applied Research in ENGINEERING SCIENCE \& TECHNOLOGY 18-20 October 2019

Budapest, Hungary

\title{
Direct-Contact Microelectrical Measurement of the Electrical Resistivity of a Solid Electrolyte Interface
}

\author{
Jun-Hyoung Park ${ }^{1}$, Yong-Soek Choi ${ }^{1}$, Hyun-Jeong Lee ${ }^{2}$, Hyung-Cheoul Shim ${ }^{3}$, Jae-Pyoung Ahn ${ }^{2}$, \\ Jae-Chul Lee ${ }^{1}$
}

1 Korea University Korea Institute of Science and Technology Korea Institute of Machinery and Materials

\begin{abstract}
Because of its effectiveness in blocking electrons, the solid electrolyte interface (SEI) suppresses decomposition reactions of the electrolyte and contributes to the stability and reversibility of batteries. Despite the critical role of SEI in determining the properties of batteries, the electrical properties of SEI layers have never been measured directly. In this paper, we present the first experimental results of the electrical resistivity of a LiF-rich SEI layer measured using a directcontact microelectrical device mounted in an electron microscope. Measurements show that the SEI layer exhibits high electrical resistivity $\left(2.3 \times 10^{5} \Omega \cdot \mathrm{m}\right)$, which is comparable with those of typical insulating materials. Furthermore, a combined technique of advanced analyses and firstprinciples calculations show that the SEI layer is mainly composed of amorphous LiF and a minute nanocrystalline $\mathrm{Li}_{2} \mathrm{CO}_{3}$ compound. The electronic origin responsible for the high
\end{abstract}




\section{2nd International Conference on Applied Research in ENGINEERING SCIENCE \& TECHNOLOGY 18-20 October 2019 Budapest, Hungary}

resistivity of the SEI layer is elucidated by calculating the band structures of various $\operatorname{Li}_{x} \mathrm{~F}$ compounds and interpreting their effects on the resistivity. This study explains why SEI can prevent the degradation of electrode materials and consumption of $\mathrm{Li}$ ions in the electrolyte and thus can be viewed as a stepping stone for developing highly stable and reversible batteries.

Keywords: Li-ion battery; solid electrolyte interface; electrical resistivity; four-point-probe technique; first-principles calculations 